\title{
Possible $Z_{2}$ phase and spin-charge separation in electron-doped cuprate superconductors
}

\author{
Tiago C. Ribeiro and Xiao-Gang Wen \\ Department of Physics, Massachusetts Institute of Technology, Cambridge, Massachusetts 02139, USA
}

(Dated: November 13, 2018)

\begin{abstract}
The $S U(2)$ slave-boson mean-field theory for the $t t^{\prime} J$ model is analyzed. The role of next-nearestneighbor hopping $t^{\prime}$ on the phase diagram is studied. We find a pseudogap phase in hole-doped (HD) materials (where $t^{\prime}<0$ ). The pseudogap phase is a $U(1)$ spin liquid (the staggered-flux phase) with a $U(1)$ gauge interaction and no fractionalization. This agrees with experiments on HD samples. The same calculation also indicates that a positive $t^{\prime}$ favors a $Z_{2}$ state with true spincharge separation. The $Z_{2}$ state that exists when $t^{\prime} \gtrsim 0.5 J$ can be a candidate for the pseudogap phase of electron-doped (ED) cuprates (if such a phase exists). The experimental situation in ED materials is also addressed.
\end{abstract}

\section{INTRODUCTION}

A complete understanding of the behavior displayed by high temperature superconducting cuprates is still lacking. In these materials, d-wave superconducting (dSC) samples are obtained upon doping the parent compounds. The latter are Heisenberg antiferromagnets. The presence of low energy properties consistent with standard BCS theory in dSC samples is also generally undisputed. However, the phase-space region intervening between the two aforementioned regimes hosts an unusual phenomenology - and a quite debated one as well. In this paper, we will adopt the point of view that the unconventional behavior in underdoped cuprates is evidence of doped Mott insulator physics. To capture the important properties of these materials, which are then controlled by the large Mott gap and the two-dimensional spin interactions in the copper-oxide planes $\frac{1}{2}$, we will use the slave-boson approach developed in Refs. 2 and 3.

In underdoped electron-doped (ED) materials the antiferromagnetic $(\mathrm{AF})$ phase is very robust. However that is not the case in their hole-doped (HD) counterparts. Instead, a pseudogap metallic normal state appears in underdoped samples. This is a paramagnetic state with a $d_{x^{2}-y^{2}}$ gaped spectral function. Reconciling the gap with the strong local AF fluctuations is not trivial though. One way to understand it is offered by the slave-boson approach to the $t J$ model. In particular, in Ref. 3 a translation symmetric pseudogap metallic state (the staggeredflux or s-flux state) was proposed. It contains spinons and holons interacting via a $U(1)$ gauge field. This state was shown to bear enhanced AF fluctuations compatible with experiments $\underline{\underline{4}}$ The $U(1)$ gauge fluctuations play a key role in the AF instability at very low doping as

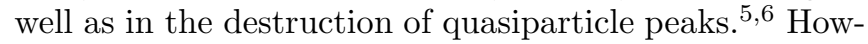
ever, as discussed in Ref. 4, the appearance of the spinpseudogap below a certain energy and the existence of well defined electronlike quasiparticles in the nodal direction above $T_{c}$ suggest that spin and charge recombine before the s-flux state reaches the dSC state.

There is a competing scenario to describe this crossover from the s-flux to the dSC phase which involves the emer- gence of an intervening state with a $Z_{2}$ gauge structure ${ }^{7}$ This state would result from the condensation of spinon bilinears $\stackrel{8.9 .10}{ }$ The dSC state would then follow from holon condensation. $Z_{2}$ spin liquids are very interesting states with true spin-charge separation. They were predicted theoretically over ten years ago ${ }^{8.9}$ Yet they lack experimental realization. The present work proposes to study the conditions favoring the emergence of these spin liquid states as well as to discuss their relevance for the cuprate superconductors.

Despite the effort to check the applicability of $Z_{2}$ spin liquids in the context of high $T_{c}$ superconductivity, so far all the experimental tests gave negative results: $\stackrel{11,12}{1}$ However, this does not imply that $Z_{2}$ states do not exist in high $T_{c}$ samples. In this paper we find that $Z_{2}$ states are indeed unlikely to exist in HD compounds. But a $Z_{2^{-}}$ linear state (i.e. with linear gapless spinon excitations) is likely to appear as the pseudogap metallic state for ED materials. In superconducting (SC) samples without $\mathrm{AF}$ order spin liquid pseudogap signatures may emerge once SC coherence is destroyed by thermal fluctuations or an external magnetic field. In the first case such a behavior is still to be reported. Experimental evidence in the magnetic field driven normal state, however, seems to be consistent with $Z_{2}$ pseudogap phenomenology 13.14

The $t J$ model has a particle-hole symmetry. In order to break it we introduce the next-nearest-neighbor (NNN) hopping $t^{\prime}$ term. Taking both signs of $t^{\prime}$ covers both ED $\left(t^{\prime}>0\right)$ and HD $\left(t^{\prime}<0\right)$ cases. In Sec. II we introduce the $S U(2)$ MF theory for the $t t^{\prime} J$ model. In Sec. III we present the resulting MF phase diagrams. For $t^{\prime}<0$ our calculations are consistent with the previous studies for $t^{\prime}=0$ where only the s-flux state was obtained. However we find that NNN hopping can stabilize a $Z_{2}$-linear state in the $t t^{\prime} J$ model for values of $t^{\prime} \gtrsim 0.5 J$. These results show that unfrustrated hopping favors fractionalized phases (Sec. IV). In Sec. $\nabla$ we establish the link between our results and some properties of ED cuprates. We draw our main conclusions in Sec. VI] 


\section{II. $S U(2)$ MF THEORY FOR THE $t t^{\prime} J$ MODEL}

The $t t^{\prime} J$ model is given by the Hamiltonian

$$
H_{t t^{\prime} J}=H_{h f}+H_{h o p}+H^{\prime}
$$

with

$$
\begin{aligned}
H_{h f} & =\sum_{<i j>} J_{i j}\left(\vec{S}_{i} \cdot \vec{S}_{j}-\frac{1}{4}\right), \\
H_{h o p} & =-\sum_{<i j>} t_{i j} \boldsymbol{P}\left(c_{i}^{\dagger} c_{j}+c_{j}^{\dagger} c_{i}\right) \boldsymbol{P}, \\
H^{\prime} & =\frac{1}{4} \sum_{<i j>} J_{i j}\left(1-n_{i} n_{j}\right),
\end{aligned}
$$

where sums are taken over pairs of sites $\langle i j\rangle$. The exchange coupling $J_{i j}$ is $J$ for $\langle i j\rangle$ nearest-neighbor (NN) sites while the hopping parameter $t_{i j}$ equals $t$ for $\langle i j\rangle$ NN sites, $t^{\prime}$ for $\langle i j\rangle \mathrm{NNN}$ sites. $\vec{S}_{i}$ is the spin on site $i, c_{i}^{\dagger}$ and $n_{i}=c_{i}^{\dagger} c_{i}$ are electron creation and occupation number operators and $\boldsymbol{P}$ projects out doubly occupied sites.

To deal with the no double occupancy constraint the electron operator is decoupled according to $c_{i \uparrow}=$ $\frac{1}{\sqrt{2}}\left(\psi_{i 1} b_{i 1}^{\dagger}+\psi_{i 2} b_{i 2}^{\dagger}\right)$ and $c_{i \downarrow}=\frac{1}{\sqrt{2}}\left(\psi_{i 2}^{\dagger} b_{i 1}^{\dagger}-\psi_{i 1}^{\dagger} b_{i 2}^{\dagger}\right)$ and the spin operators are expressed as $\vec{S}_{i}=\frac{1}{2} f_{i \alpha}^{\dagger} \vec{\tau}_{\alpha \beta} f_{i \beta}$ where the $S U(2)$ doublets $\Psi_{i}^{\dagger}=\left[\psi_{i 1}^{\dagger} \psi_{i 2}^{\dagger}\right]=\left[f_{i 1}^{\dagger} f_{i 2}\right]$ and $h_{i}^{\dagger}=\left[b_{i 1}^{\dagger} b_{i 2}^{\dagger}\right]$ represent spinons (fermionic particles carrying spin $\frac{1}{2}$ ) and holons (bosonic particles carrying unit charge) respectively and $\vec{\tau}$ are the Pauli matrices. These operators were introduced in Ref. 3 to keep the $S U(2)$ gauge structure of $H_{t t^{\prime} J}$ even away from half-filling. Parton operators introduce unphysical degrees of freedom which are projected out by implementing the constraint $\vec{Q}_{i} \equiv \Psi_{i}^{\dagger} \vec{\tau} \Psi_{i}+h_{i}^{\dagger} \vec{\tau} h_{i}=0$ (i.e. physical states are $S U(2)$ singlets on every site). Introducing a Lagrange multiplier per lattice site $\left(\vec{a}_{0 i}\right)$ the problem is reduced to one of lattice gauge theory $\stackrel{3}{-}$ Performing a Hartree-Fock type of decoupling of $H_{h f}$ and $H_{h o p}$ (neglecting $H^{\prime}$ for the time being) and implementing the projection constraint at $\mathrm{MF}$ level only, the following MF Hamiltonian is obtained:

$$
\begin{aligned}
H_{M F} & =\frac{3}{16} \sum_{<i j>} J_{i j} \operatorname{Tr}\left(U_{i j} U_{j i}\right)+\frac{1}{2} \sum_{<i j>} t_{i j} \operatorname{Tr}\left(U_{i j} B_{j i}\right) \\
& +\sum_{i} \vec{a}_{0 i} .\left(\Psi_{i}^{\dagger} \vec{\tau} \Psi_{i}+h_{i}^{\dagger} \vec{\tau} h_{i}\right)-\mu_{b} \sum_{i} h_{i}^{\dagger} h_{i} \\
& -\frac{1}{2} \sum_{<i j>} t_{i j}\left(h_{i}^{\dagger} U_{i j} h_{j}+\text { h.c. }\right) \\
& -\sum_{<i j>}\left[\Psi_{i}^{\dagger}\left(\frac{3}{8} J_{i j} U_{i j}+\frac{1}{2} t_{i j} B_{i j}\right) \Psi_{j}+\text { h.c. }\right]
\end{aligned}
$$

where $\mu_{b}$ is the chemical potential of holons.

Despite ignoring gauge fluctuations, we expect MF theory to account for some qualitatively right features. Indeed, we have the following: i) The energetics of the model is correctly captured at MF level. This fact is suggested by the $S U(2)$ slaveboson MF theory phase diagram for the $t J$ model - it includes the pseudogap regime (s-flux state) that turns into the dSC state as holons become coherent, as well as the strange-metal and Fermi-liquid regimes. ${ }^{3}$ In particular, the pseudogap metallic state was predicted by the slaveboson approach prior to experimental observation; 15

ii) The nature of fluctuations in each phase is also consistent with experiments. For instance, the $U(1)$ gauge structure in the s-flux state accounts for the spin excitation spectrum in underdoped HD materials ${ }^{4}$ It also leads to the lack of well defined quasiparticles ${ }^{\frac{5}{5}}$ In the strangemetal regime $S U(2)$ gauge fluctuations are responsible for the observed non-Fermi liquid behavior;

iii) $\mathrm{MF}$ states have properties that survive projection even in the presence of long range $U(1)$ gauge fields. Take the s-flux state, which includes both staggered current and d-wave pairing fluctuations, as an example. Remarkably, in Ref. 16 staggered vorticity correlations were reported to emerge from Gutzwiller projecting the dSC wave function. There is also numerical evidence that $Z_{2}$ MF states lead to fractionalized phases after performing Gutzwiller projection. 17

\section{MF PHASE DIAGRAMS}

To implement the self-consistent MF equations for the $t t^{\prime} J$ model, the uniform ansatz considered in Ref. 3 must be extended to include the NNN MF parameters $U_{i, i+\hat{x} \pm \hat{y}}$. Here we only consider translation invariant ansatsë which do not break any symmetry. In our MF calculation we use the following ansatz which properly comprises both the s-flux and dSC states:

$$
\begin{aligned}
U_{i, i+\hat{x}} & =\chi \tau^{3}+\eta \tau^{1}, \\
U_{i, i+\hat{y}} & =\chi \tau^{3}-\eta \tau^{1}, \\
U_{i, i+\hat{x} \pm \hat{y}} & =\gamma \tau^{3} \\
a_{0}^{3} & \neq 0 .
\end{aligned}
$$

Depending on the values of $\chi, \eta$, and $\gamma$, the above ansatz can describe phases with the same symmetry but different quantum orders ${ }^{10}$ These phases may contain $S U(2)$, $U(1)$, or $Z_{2}$ gauge fluctuations. Some phases display a large Fermi surface while others have only Fermi points. These different MF states and their labels are summarized in Table $\amalg$

The MF phase diagrams are determined for $t=3 \mathrm{~J}$ and $t^{\prime}$ between $-1.5 \mathrm{~J}$ and $1.5 \mathrm{~J}$. These values are representative of the cuprates (note that $t^{\prime}$ is close to $J$ for ED samples). Holons may condense at nonzero temperatures due to interactions or to the projection constraint (as proposed in Ref. 3). However, at MF level holons are free and conventional holon condensation only occurs at $T=0$. In our calculations we take $T>0$ and, hence, only consider the unconventional mechanism. 


\begin{tabular}{|c|c|c|c|c|}
\hline \hline $\begin{array}{c}\text { MF } \\
\text { phase }\end{array}$ & $\begin{array}{c}\text { Gauge } \\
\text { structure }\end{array}$ & \multicolumn{3}{|c|}{$\begin{array}{c}\text { MF } \\
\text { parameters }\end{array}$} \\
\hline s-flux (sf) & $U(1)$ & $\chi \neq \eta$ & $\gamma=a_{0}^{3}=\rho=0$ & $\chi, \eta \neq 0$ \\
\hline $\mathrm{Z} 2$ & $Z_{2}$ & $\chi \neq \eta$ & $\chi, \eta, \gamma, a_{0}^{3} \neq 0$ & $\rho=0$ \\
\hline $\mathrm{dSC}$ & $Z_{1}$ & $\chi \neq \eta$ & $\chi, \eta, \gamma, a_{0}^{3}, \rho \neq 0$ \\
\hline $\mathrm{U} 1$ & $U(1)$ & $\eta=\rho=0$ & $\chi, \gamma, a_{0}^{3} \neq 0$ \\
\hline $\mathrm{FL}$ & $Z_{1}$ & $\eta=0$ & $\chi, \gamma, a_{0}^{3}, \rho \neq 0$ \\
\hline $\mathrm{uRVB}$ & $S U(2)$ & $\chi \neq 0$ & $\eta=\gamma=a_{0}^{3}=\rho=0$ \\
\hline$\pi$-flux $(\pi \mathrm{f})$ & $S U(2)$ & $\chi=\eta \neq 0$ & $\gamma=a_{0}^{3}=\rho=0$ \\
\hline \hline
\end{tabular}

TABLE I: Labels for MF phases. $\rho$ is the density of condensed holons. All other parameters are defined in the main text
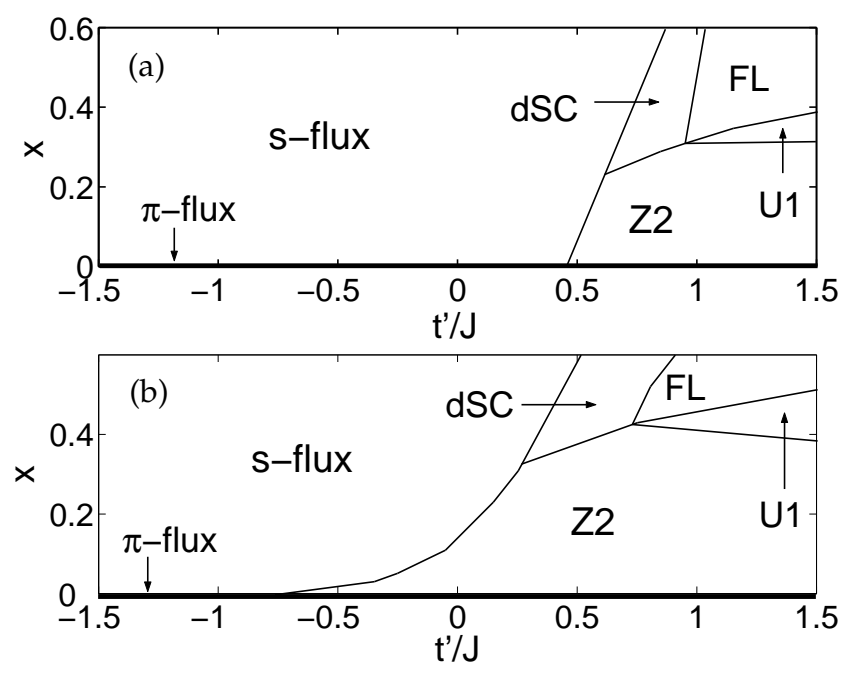

FIG. 1: (a) $\left(t^{\prime}, \mathrm{x}\right)$ phase diagram for $H_{M F}$ at $T=0^{+}$. (b) $\left(t^{\prime}, \mathrm{x}\right)$ phase diagram at $T=0^{+}$after including $H^{\prime}$ and $H^{\prime \prime}$

The resulting $\left(t^{\prime}, x\right)$ phase diagram for $T=0^{+}$is shown in Fig प(a). For $t^{\prime} \lesssim 0.5 J$ the s-flux state survives all the way to $T=0^{+}-$it becomes the dSC state for $T=0$. However, for $t^{\prime} \gtrsim 0.5 J$ new MF states are obtained. In the underdoped regime, where the $S U(2)$ theory was proposed to be relevant, the $\mathrm{Z} 2$ phase emerges. When $T=0$ holons condense in the band bottom and the $\mathrm{Z} 2$ state changes into the dSC phase. For high enough doping we find the dSC state even at nonzero temperature - resulting from projection constraint driven condensation. As the pseudogap closes $(\eta \rightarrow 0)$ a state with $U(1)$ gauge structure and gapless spinons arises (U1). Upon holon condensation it becomes a Fermi liquid (FL).

For $t^{\prime} \lesssim 0.5 J$ we obtain the $(x, T)$ phase diagram reported previously $\frac{3}{3}$ The MF finite temperature phase diagrams are illustrated for $t^{\prime}=0.8 \mathrm{~J}$ and $t^{\prime}=1.2 \mathrm{~J}$ in Figs. 22(a) and 2(b) respectively. As, by construction, the $\mathrm{AF}$ phase is absent, the $Z_{2}$-linear state appears as the dominant phase at low doping. ED samples can have three different normal metallic states: (a) a Z2 state with a $d$-wave pseudogap, true spin-charge separation and a
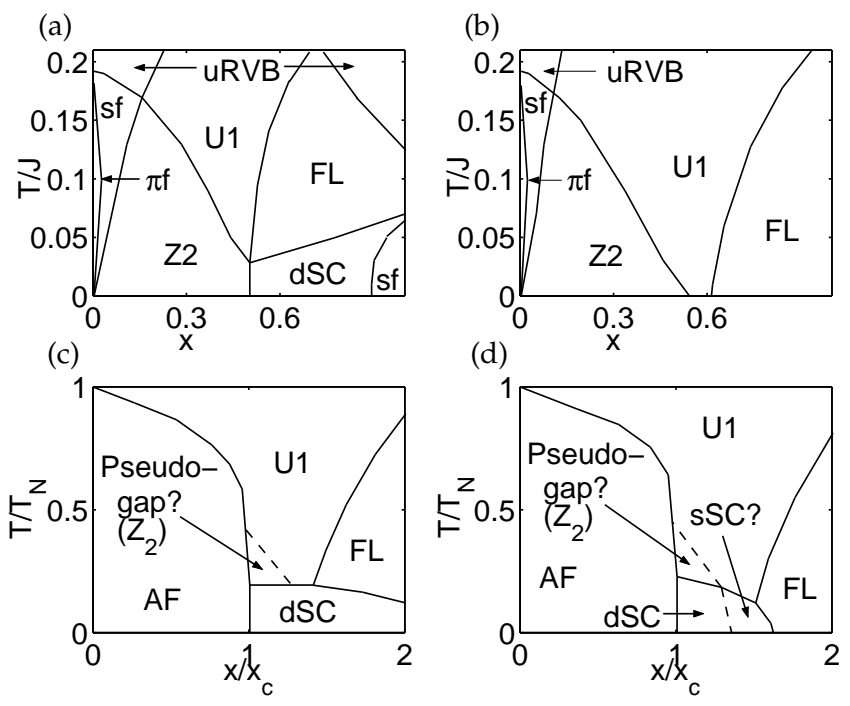

FIG. 2: (x,T) MF phase diagrams for (a) $t^{\prime}=0.8 J$ and (b) $t^{\prime}=1.2 J$. In (c) and (d) qualitative phase diagrams, based on the MF results from (a) and (b) respectively, are proposed. These include the experimentally observed AF phase and finite temperature holon condensed phase due to holon interaction. The dashed line describes the transition into the Z2 state in case it is not completely absorbed by the AF phase. Above $T_{c}$ the Z2 state describes pseudogap behavior. In (d) this transition may describe the reported transition from dSC to an extended s-wave SC (sSC) phase in $\mathrm{Pr}_{2-x} \mathrm{Ce}_{x} \mathrm{CuO}_{4-y}$ and $\mathrm{La}_{2-x} \mathrm{Ce}_{x} \mathrm{CuO}_{4-y}$ (Ref. 18).

nontrivial topological order, ${ }^{7.8 .9}$ (b) a strange metal (the U1 phase) with a large Fermi surface and a $U(1)$ gauge interaction, and (c) a FL phase.

The above discussion of the MF phase diagrams is qualitatively correct even after including the dropped $H^{\prime}$ term. In terms of spinons and holons, $H^{\prime}$ has the form

$$
\begin{aligned}
H^{\prime}= & -\frac{J}{2} \sum_{i}\left(\Psi_{i}^{\dagger} \vec{\tau} \Psi_{i}\right)\left(h_{i}^{\dagger} \vec{\tau} h_{i}\right) \\
& -\frac{J}{16} \sum_{i} \sum_{\hat{u}=\hat{x}, \hat{y}} h_{i}^{\dagger} h_{i} h_{i+\hat{u}}^{\dagger} h_{i+\hat{u}}+O\left(x^{3}\right),
\end{aligned}
$$

(which is $\propto x^{2}$ at the MF level). While the second term correctly describes the attraction between holons resulting from the background with AF fluctuations, the first one should have the opposite sign to correctly account for the attraction between spinons and holons caused by gauge fluctuations. Hence, $H^{\prime}$ should be considered together with an effective contribution arising from the gauge interaction. At MF level the constraint is implemented only globally, i.e. $\left\langle\sum_{i} \vec{Q}_{i}\right\rangle=0$. Including the following effective interaction, $H^{\prime \prime}=\frac{3 J}{16} \sum_{i, \hat{u}=\hat{x}, \hat{y}}\left(\vec{Q}_{i}-\right.$ $\left.\vec{Q}_{i+\hat{u}}\right)^{2}$, the fluctuations of $\left(\vec{Q}_{i}\right)^{2}$ away from zero are reduced. The prefactor in $H^{\prime \prime}$ is such that the exchange constant is renormalized to $J_{\text {eff }}=1.5 \mathrm{~J}$ (note that Gutzwiller projection is known to increase the effective exchange constant ${ }^{19}$ ). $H^{\prime \prime}$ also makes holons more 
massive. Including $H^{\prime}$ and $H^{\prime \prime}$ at the MF level extends the region where the Z2 state emerges (Fig प(b)). Hence gauge fluctuations stabilize the Z2 phase. As expected, projection has a smaller effect in this phase.

The above results support that the $Z_{2}$-linear spin liquid introduced in Sec. [I is a competitive state for higher values of $t^{\prime}$ and low doping levels. In particular, the lower bound $t^{\prime} \gtrsim 0.5 \mathrm{~J}$ makes this state relevant for ED cuprates.

\section{SPIN-CHARGE SEPARATION: THE ROLE OF $t^{\prime}$}

The $S U(2)$ slave-boson approach is designed to address the low doping regime. As it is clear from Fig 1 for low doping there are two dominant phases, namely, the s-flux state and the Z2 state. The former becomes the latter as $t^{\prime}$ is increased above a certain critical value. This parameter driven transition links two states with the same symmetries (they both describe totally symmetric spin liquids). However they have different quantum orders 10 This statement can be made more explicit by emphasizing the quite different nature of their low-energy effective behavior.

In the s-flux state massless Dirac fermions (spinons) and charged bosons (holons) interact with a long-range $U(1)$ gauge field. The gapless nature of these interacting excitations is protected by the quantum order $\stackrel{10}{10}$ Still, the gauge field has a drastic effect on the nature of the excitations - this state is in fact a manifestation of a two-dimensional Luttinger liquid ${ }^{4,5.6}$ which has no well defined quasiparticles.

On the other hand, in the Z2 state the gauge interaction becomes short-ranged. Hence, the (linearly dispersing) spinons and holons are well defined quasiparticles. For that reason there is true spin-charge separation. The remnant discrete $Z_{2}$ gauge structure reflects the topological order of the state. $\underline{\underline{9}}$ This topological order is related to the ground-state degeneracy of the system when it is embedded in a manifold with nontrivial topology. Such degenerate ground-states are locally similar but have different global (topological) properties.

The different gauge structure in the two states $(U(1)$ and $Z_{2}$ ) naturally gives rise to qualitatively distinct spectra for the collective modes. Indeed, the transition is accompanied by the opening of a gap in the fluctuations around the MF saddle-point 20

Physically, the transition from the s-flux state to the Z2 state corresponds to the emergence of coherent diagonal (intrasublattice) charge carrier hopping - i.e. $\left\langle c_{i}^{\dagger} c_{i+\hat{u}}+c_{i+\hat{u}}^{\dagger} c_{i}\right\rangle$, with $\hat{u}= \pm \hat{x} \pm \hat{y}$, becomes nonzero. It results from a combination of two factors: (a) increasing $t^{\prime}$ unfrustrates diagonal hopping and (b) electron/hole intrasublattice hopping is not frustrated and does not frustrate the background AF correlations (unlike intersublattice hopping). These play a crucial role in the dynamics of charge carriers (and of spin degrees of freedom as well).

In the s-flux state intrasublattice hopping is depleted by the negative $t^{\prime}$. As charge carriers hop between different sublattices they interact strongly with the background AF fluctuations. Hence charge carrier motion and $\mathrm{AF}$ correlations frustrate each other. That is the motive underlying the staggered current and staggered chiral spin correlations ${ }^{21}$ The presence of such correlations in the context of the $t J$ model was reported in exact diagonalization studies ${ }^{22}$ Moreover, charge carrier density and vorticity correlations are proportional to each other ${ }^{16.22}$ The emerging picture consists of charge carriers of opposite vorticity attracting each other in a background of staggered chiral spin fluctuations. This attraction eventually induces dSC coherence.

In the Z2 state charge carriers hop coherently along the diagonals and the staggered current correlations decrease. In particular, the energy difference between the dSC and staggered current states is seen to increase with $t^{\prime}$. Therefore, staggered current fluctuations are reduced in the Z2 state. Exact diagonalization studies in doped $t t^{\prime} J$ ladders show the same trend - i.e. staggered currents are stabilized by decreasing $t^{\prime} 2^{23}$ According to our calculations, spinon pairing $(\eta)$ is also reduced by the emergence of coherent diagonal hopping (therefore, charge carrier pairing may be expected to decrease as well). This is further consistent with the fact that the $Z_{2}$-vortex gap is physically connected to the reduction of staggered chiral spin fluctuations ${ }^{20.24}$

Topological order in the $\mathrm{Z} 2$ state is associated with the intrasublattice hopping parameter $\gamma$. Studies of Gutzwiller-projected wave functions in Ref. 17 further corroborate the importance of the NNN hopping term (i.e. a non-vanishing $\gamma$ ) in realizing true fractionalization. Consequently, for underdoped samples, the $Z_{2}$-vortex gap in this fractionalized metallic pseudogap phase is set by $\gamma$ which is $\propto x$. This is qualitatively different from the suggestion of Senthil and Fisher where the $Z_{2}$-vortex gap is set by the pseudogap scale $(\eta), 12$

The arguments above support and delineate how the parameter $t^{\prime}$ induces spin-charge separation. However, to fit experimental band structure, an extra hopping term $\left(t^{\prime \prime}\right)$ must be included 27 This requires an extension of the ansatsë considered in our analysis. Nevertheless, in a tight-binding model with $t>0, t^{\prime}>0$ and $t^{\prime \prime}<0$ (as it is the case for ED cuprates), $t^{\prime \prime}$ is seen to frustrate hopping along the lattice bonds while unfrustrating hopping along the diagonals - in this sense enhancing the effect of $t^{\prime}$.

\section{APPLICATION TO ED CUPRATES}

As mentioned before, in ED cuprates the AF state is very stable. Such stability can be addressed within the $t t^{\prime} J$ model framework. Indeed, an increasing $t^{\prime}$ unfrustrates intrasublattice hopping and renders the charge carriers less effective in frustrating the background $\mathrm{AF}$ correlations ${ }^{28}$ However, our MF results suggest that, 
if $\mathrm{AF}$ order can be destroyed by tuning parameters in the Hamiltonian, the resulting spin liquid may contain a pseudogap and correspond to the Z2 state.

A distinctive experimental feature of the $\mathrm{Z} 2$ phase is the presence of a $d$-wave pseudogap 29 Well, in the different $\mathrm{Nd}_{2-x} \mathrm{Ce}_{x} \mathrm{CuO}_{4-y}, \mathrm{Pr}_{2-x} \mathrm{Ce}_{x} \mathrm{CuO}_{4-y}(\mathrm{PCCO})$, and $\mathrm{La}_{2-x} \mathrm{Ce}_{x} \mathrm{CuO}_{4-y}$ (LCCO) SC thin films, the SC gap $(\sim 5-10 \mathrm{meV})$ is reported to survive in the magnetic field driven normal state 13 The value of the normal state gap is comparable to the SC gap and decreases with doping. Moreover, the low temperature normal state in PCCO SC crystals was reported to violate the Wiedemann-Franz law 14 This provides evidence for a non-Fermi liquid state compatible with spin-charge separation. According to our MF results, the aforementioned normal state may realize the Z2 state. On the other hand, if the Z2 state is to exist above $T_{c}$ it probably only covers a small fraction of the phase diagram due to the robust AF phase (Figs 2(c) and 2(d)) 33 $^{33}$

Despite still disputed, there are a number of experiments reporting dSC order in ED samples ${ }^{34}$ In our picture, the $d_{x^{2}-y^{2}}$ gap in the SC phase is inherited from spinon pairing in the Z2 state. Recently, however, a SC pairing symmetry transition across optimal doping, from d-wave to extended s-wave, was reported in both LCCO and $\mathrm{PCCO}, 18$ We suggest the extended s-wave pairing may result in the FL state (Fig 2/d)) from exchange of collective modes - as the interaction between the Fermi sea and the massive gauge-fields raises an instability in the s-wave pairing channel. ${ }^{35}$ We remark that this mech- anism is unrelated to the one underlying dSC.

\section{CONCLUSIONS}

In this paper we use a self-consistent $S U(2)$ slave-boson approach to study the role of NNN hopping in the context of the $t t^{\prime} J$ model. We find that $t^{\prime}$ induces a transition between the s-flux state (an algebraic spin liquid ${ }^{4.5}$ ) and the Z2 state (a spin liquid with true spin-charge separation). This transition is interpreted as the emergence of coherent intrasublattice hopping in the Z2 state. The parameter $t^{\prime}$ is also found to decrease staggered current and staggered chiral spin fluctuations, as well as to reduce pairing between charge carriers.

The range of parameters involved leads us to propose that a pseudogap metallic state in ED cuprates is likely to be described by a $Z_{2}$-linear phase. In contrast, the pseudogap state for HD samples is found to be described only by the s-flux phase with $U(1)$ gauge interaction.

Realizing fractionalized states in real systems is a major challenge. The present work suggests that the search for a pseudogap metallic state in ED cuprate samples may achieve such a goal.

We would like to acknowledge many discussions with W. Rantner. TCR was partially supported by the Praxis XXI Grant No. BD/19612/99 (Portugal). TCR and XGW were also supported by NSF Grant No. DMR-0123156 and by NSF-MRSEC Grant No. DMR-02-13282.
1 P. W. Anderson, Science 235, 1196 (1987).

2 G.Baskaran, Z. Zou and P. W. Anderson, Solid State Commun. 63, 973 (1987); G. Baskaran and P. W. Anderson, Phys. Rev. B 37, 580 (1988); I. Affleck, Z. Zou, T. Hsu and P. W. Anderson, Phys. Rev. B 38, 745 (1988).

3 X.-G. Wen and P. A. Lee, Phys. Rev. Lett. 76, 503 (1996); P. A. Lee, N. Nagaosa, T.-K. Ng, X.-G. Wen, Phys. Rev. B 57, 6003 (1998).

${ }^{4}$ W. Rantner and X.-G. Wen, cond-mat/0201521.

${ }^{5}$ W. Rantner and X.-G. Wen, Phys. Rev. Lett. 86, 3871 (2001).

6 M. Franz and Z. Tesanovic, Phys. Rev. Lett. 87, 257003 (2001).

7 T. Senthil and M. P. A. Fisher, Phys. Rev. B 62, 7850 (2000).

8 N. Read and S. Sachdev, Phys. Rev. Lett. 66, 1773 (1991).

9 X.-G. Wen, Phys. Rev. B 44, 2664 (1991).

10 X.-G. Wen, Phys. Rev. B 65, 165113 (2002).

11 J. C. Wynn, D. A. Bonn, B. W. Gardner, Y. J. Lin, R. Liang, W. N. Hardy, J. R. Kirtley and K. A. Moler, Phys. Rev. Lett. 87, 197002 (2001); D. A. Bonn, J. C. Wynn, B. W. Gardner, Y. J. Lin, R. Liang, W. N. Hardy, J. R. Kirtley and K. A. Moler, Nature 414, 887 (2001).

12 T. Senthil and M. P. A. Fisher, Phys. Rev. B 64, 214511 (2001); T. Senthil and M. P. A. Fisher, Phys. Rev. Lett. 86, 292 (2001).

13 S. Kleefisch, B. Welter, A. Marx, L. Alff, R. Gross and
M. Naito, Phys. Rev. B 63, 100507 (2001); A.Biswas, P. Fournier, V. N. Smolyaninova, R. C. Budhani, J. S. Higgins and R. L. Greene, Phys. Rev. B 64, 104519 (2001); L. Alff, Y. Krockenberger, B. Welter, M. Schonecke, R. Gross, D. Manske and M. Naito, Nature 422, 698 (2003).

14 R.W.Hill, C. Proust, L. Taillefer, P. Fournier and R. L. Greene, Nature 414, 711 (2001).

15 G. Kotliar and J. Liu, Phys. Rev. B 38, 5142 (1988).

16 D. A. Ivanov, P. A. Lee and X.-G. Wen, Phys. Rev. Lett. 84, 3958 (2000).

17 D. A. Ivanov and T. Senthil, Phys. Rev. B 66, 115111 (2002).

18 A. Biswas, P. Fournier, M. M. Qazilbash, V. N. Smolyaninova, Hamza Balci and R. L. Greene, Phys. Rev. Lett. 88, 207004 (2002); J.A.Skinta, M. S. Kim, T. R. Lemberger, T. Greibe and M. Naito, Phys. Rev. Lett. 88, 207005 (2002).

19 F. C. Zhang, C. Gros, T. M. Rice and H. Shiba, Supercond. Sci. Technol. 1, 36 (1988).

20 T. C. Ribeiro and X.-G. Wen, unpublished.

${ }^{21}$ X.-G. Wen, F. Wilczek and A. Zee , Phys. Rev. B 39, 11413 (1989); P. A. Lee and N. Nagaosa, Phys. Rev. B 46, 5621 (1992).

22 P. W. Leung, Phys. Rev. B 62, R6112 (2000).

${ }^{23}$ K. Tsutsui, D. Poilblanc and S. Capponi, Phys. Rev. B 65, 020406 (2002).

24 Results based on numerical calculations on ladder systems show that hole binding is favored by an increasing $t^{\prime}$ (Refs. 
23 and 25). The sensitiveness to the effective dimensionality of the system under analysis is reflected in Fig.12 of Ref. 26. In Ref. 25 some qualitative arguments on small clusters were put forth to motivate the aforementioned results. These differ from the picture we propose. In the present approach the pairing parameter $\eta$ (which is not the superconducting order parameter obtained from the electron d-wave pair field correlation function) emerges in the spin sector due to the highly frustrated antiferromagnetic background with non-local correlations. As suggested above such pairing is enhanced by staggered chiral spin fluctuations. Our results support that increasing $t^{\prime}$ reduces these fluctuations and facilitates single charge carrier intrasublattice hopping.

25 S. R. White and D. J. Scalapino, Phys. Rev. B 60, R753 (1999); G. B. Martins, J. C. Xavier, L. Arrachea and E. Dagotto, Phys. Rev. B 64, 180513(R) (2001).

26 R. J. Gooding, K. J. E. Vos and P. W. Leung, Phys. Rev. B 49, 4119 (1994).

27 C. Kim, P. J. White, Z.-X. Shen, T. Tohyama, Y. Shibata, S. Maekawa, B. O. Wells, Y. J. Kim, R. J. Birgeneau and M. A. Kastner, Phys. Rev. Lett. 80, 4245 (1998); K. Tsutsui, T. Tohyama and S. Maekawa, Phys. Rev. Lett. 83, 3705 (1999).

28 T. Tohyama and S. Maekawa, Phys. Rev. B 49, 3596 (1994); R. J. Gooding, K. J. E. Vos, P. W. Leung, Phys.
Rev. B 50, 12866 (1994).

29 There is a large gap, evolving from $\sim 400 \mathrm{meV}$ for $x=0.04$ to $\sim 100 \mathrm{meV}$ for $x=0.15$, in the stable AF phase in ED samples of $\mathrm{Nd}_{2-x} \mathrm{Ce}_{x} \mathrm{CuO}_{4-y}$ according to ARPES (Ref. 30) and optical conductivity (Ref. 31) measurements. It results from a $(\pi, \pi)$ scattering mechanism (Ref. 32) and is not the pseudogap in the Z2 state.

30 N. P. Armitage, F. Ronning, D. H. Lu, C. Kim, A. Damascelli, K. M. Shen, D. L. Feng, H. Eisaki, Z.-X. Shen, P. K. Mang, N. Kaneko, M. Greven, Y. Onose, Y. Taguchi and Y. Tokura, Phys. Rev. Lett. 88, 257001 (2002).

31 Y. Onose, Y. Taguchi, K. Ishizaka and Y. Tokura, Phys. Rev. Lett. 87, 217001 (2001).

32 N. P. Armitage, D. H. Lu, C. Kim, A. Damascelli, K. M. Shen, F. Ronning, D. L. Feng, P. Bogdanov, Z.-X. Shen, Y. Onose, Y. Taguchi, Y. Tokura, P. K. Mang, N. Kaneko and M. Greven, Phys. Rev. Lett. 87, 147003 (2001).

33 As noted above, in the absence of the Z2 pseudogap regime above $T_{c}$ our MF results indicate that the normal state can be described by either a strange metal (U1 phase) or a Fermi liquid.

34 C. C. Tsuei and J. R. Kirtley, Rev. Mod. Phys. 72, 969 (2000).

35 P. A. Lee, Phys. Rev. Lett. 63, 680 (1989). 